\title{
ASCA OBSERVATION OF NGC1333 STAR FORMING REGION
}

M. ITOH AND H. FUKUNAGA

Kobe University, Tsurukabuto, Nada, Kobe 657 Japan

K. KOYAMA AND Y. TSUBOI

Department of Physics, Graduate School of Science

Kyoto University, Sakyo-ku, Kyoto 606-01 Japan

S. YAMAUCHI

Faculty of Humanities and Social Sciences, Iwate University

Ueda, Morioka, Iwate 020 Japan

N. KOBAYASHI AND M. HAYASHI

National Astronomical Observatory

Osawa, Mitaka, Tokyo, 181 Japan

AND

S. UENO

Department of Physics and Astronomy, University of Leicester Leicester, UK

The region south of the reflection nebula NGC1333 in Perseus is an active star forming region including numerous Herbig-Haro objects and at least 5 protostar candidates with molecular outflows and far-infrared emission. It has been actively studied in various wave bands (e.g. Aspin et al 1994 and references therein). We observed this region with $A S C A$ with the primary objective to detect X-rays from the protostars embedded deep in the molecular cloud.

The X-ray images obtained by $A S C A$ are shown in Fig.1. While the soft $\mathrm{X}$-ray image is dominated by 4 or 5 point sources, about $15 \mathrm{X}$-ray sources were detected with the statistical significance higher than $5 \sigma$ in the hard band. The positions of the protostar candidates are plotted in the figure. There are two cases of possible coincidence of the X-ray source position with the protostar candidate within the positional error circle: one is for SVS13(IRAS 3), and the other is for IRAS 2. However, these sources were detected by ROSAT/HRI observations with superior positional accuracy, and Preibisch (1997) identified them with other nearby sources. 


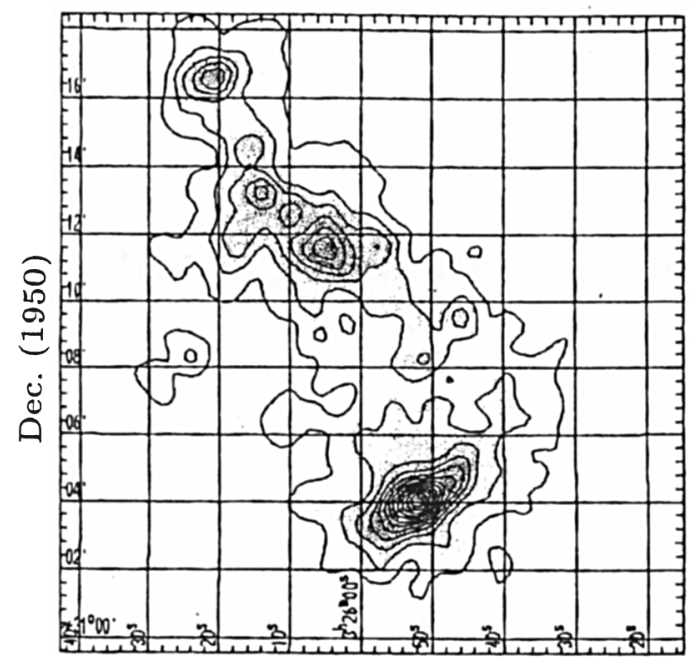

R.A. (1950)

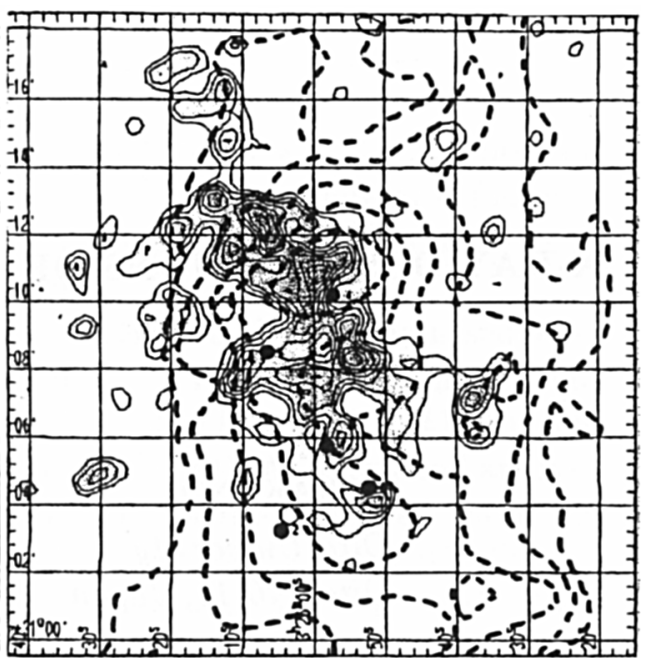

R.A. (1950)

Figure 1. X-ray inages of the NGC1333 south in soft (0.5-2 keV: left) and hard (4-10 $\mathrm{keV}$ : right) energy bands. In the hard band image, positions of the protostar candidates are indicated with filled circles, and the contours of the $\mathrm{C}^{18} \mathrm{O}$ column density (broken lines) are overlaid.

Overall distribution of the hard X-ray emission follows the high column density region traced by the $\mathrm{C}^{18} \mathrm{O}$ emission (Warin et al. 1996) as shown in Fig.1. Since it is difficult to extract spectrum of the individual sources with the point spread function of the $A S C A$ telescope, we examined the $\mathrm{X}$ ray spectrum of a region centered at $\left(3^{h} 26^{m} 10^{s}, 31^{\circ} 08^{\prime} 00^{\prime \prime}\right)$ with the radius of 2 arcmin which includes some of the hard sources and is free from the contamination from the sources visible in the soft band. The spectrum can be fit with thermal bremsstrahlung of $k T \sim 4-13 k e V$ ( $90 \%$ confidence error) and the absorption of $N_{H} \sim 10^{22} \mathrm{~cm}^{-2}$. The X-ray luminosities of the detected hard sources are estimated to be $\sim 10^{30} \mathrm{erg}^{-\mathrm{sec}^{-1}}$ assuming the distance of $350 \mathrm{pc}$. These results imply that the $A S C A$ sources are young stellar objects embedded in the molecular cloud. Significant time variation was observed for some of the detected sources, but no flare-like event was detected.

In conclusion, we did not detected X-rays from the known protostar candidates, but we detected X-rays from new young stellar objects. Evolutionary stages of these objects are to be studied by future observations.

\section{References}

Aspin, C., Sandell, G., Russel, A.P.G., 1994, A\&AS 106,165

Cernis, K.,1990, ApSS, 166,315

Preibisch, T. 1997, A\&A, 324, 690

Warin, S., et al, 1996, A\&A, 306, 935 